\title{
A Novel Robust Hexarotor Capable of Static Hovering in Presence of Propeller Failure
}

\author{
Elgiz Baskaya $^{1 \star}$, Mahmoud Hamandi $^{2 \star}$, Murat Bronz ${ }^{1}$, Antonio Franchi ${ }^{3,2}$
}

\begin{abstract}
This paper presents a novel open source design of the Y-shaped hexarotor Unammend Aerial Vehicle (UAV), and proves both in theory and real experiments its robustness to the failure of any of its propellers. An intuitive geometrical interpretation of UAV static hovering ability is presented, through which the robustness of different coplanar/collinear hexarotor designs is analyzed. Following the presented geometrical interpretation, we also show the conditions that allow the Star-shaped hexarotor to be robust to the failure of some of its propellers, while showing its incapability to static hover in the case of the failure of any of its propellers. Finally, the efficiency of the Y-shaped and Star-shaped hexarotors are tested experimentally, and conclusions on the advantages and disadvantages of the two designs are drawn.
\end{abstract}

Index Terms-Aerial Systems: Mechanics and Control, Aerial Systems: Applications, Failure Detection and Recovery

\section{INTRODUCTION}

$\mathbf{U}$ NMANNED Aerial Vehicles (UAV)s are now widely used in research and industry thanks to their versatility and large field of applications, such as aerial physical interaction [1], [2] and human-robot collaboration [3], [4]. Among the variety of UAV designs discussed in the literature [5], it seems that in the above applications, quadrotors, hexarotors, and octorotors are the most commonly used; this is most commonly due to their flight efficiency as compared to more compex UAVs, in addition to the ease and low cost of their production.

For the above applications, and any similar application that requires UAVs to hover near other objects or humans, it is of paramount importance, for safety and reliability, that the multirotor is designed to withstand at least a single propeller failure, and precisely land after the fault. It has been shown that it is possible to still fly multi-rotors with less than six propellers (e.g., quadrotors) after the loss of one or more propellers [6]. In such cases, however, the platform is no longer able to statically hover, i.e., to keep a zero translational and angular velocity, where these platforms start to loiter and spin at an uncontrolled speed, while only their average location can be controlled in practice (referred to as dynamic hovering). This is an acceptable compromise if the multirotor flies in an open

$\star$ The two authors contributed equally to the work presented in this paper.

${ }^{1}$ Ecole Nationale de l'Aviation Civile, Toulouse, France murat.bronz@enac.fr, elgiz.baskaya@enac.fr

${ }^{2}$ LAAS-CNRS, Université de Toulouse, CNRS, Toulouse, France, mahmoud.hamandi@laas.fr, antonio.franchielaas.fr

${ }^{3}$ Robotics and Mechatronics lab, Faculty of Electrical Engineering, Mathematics \& Computer Science, University of Twente, Enschede, The Netherlands a.franchi@utwente.nl

This work was partially funded by the European Commission under the project H2020 AERIAL-CORE (EC 871479) \& by the ENAC Groupe ADP Sopra Steria Research Chair on Drone Systems. space and if its exteroceptive perception system can still work in such highly dynamic conditions. However, in the previously mentioned working conditions, dynamic hovering is not an acceptable fall-back solution.

Recently [7] have introduced a stronger notion of robustness to propeller failure, as the capability to statically hover after the loss of any of the multi-rotor propellers. In order to meet an acceptable safety standard, this property is a required one for any platform flying in a critical environment. It has been shown that, in order to achieve static hovering robustness for a platform with alternating propeller rotation direction, six propellers are minimally required [7]. Furthermore, surprisingly, it has been shown that the standard and widespread Star-shaped hexarotors (see Fig. 1-right) are not robust in such sense. This counterintuitive phenomenon can be seen for example in [8], [9] where simulations and experiments show that the best a model predictive controller is able to achieve in such case is dynamic hovering, even if five propellers are still available. ${ }^{1}$ Similar outcomes are obtained from other commercially available platforms ${ }^{2}$.

The mathematical reasons for such vulnerability have been deeply analyzed in [7], where it is explained that in order to achieve robust hexarotor platforms one possibility is to use a Star-shaped platform with tilted propellers [10], [11]. Exploiting this fact, two new prototypes have been built. One prototype in [12] is a Star-shaped hexarotor platform where one of the propellers can be quickly titled via a servomotor in case of the loss of any of its propellers in order to recover static hoverability. Another prototype, built and experimentally demonstrated in [7], is a Star-shaped platform with constantly tilted propellers. The robustness of both prototypes have been shown in real experiments.

Another way to obtain robustness, also illustrated theoretically in [7] is to use a non-Star-shaped hexarotor, like, e.g., the Y-shaped hexarotor [7] depicted in Fig. 1-left. Such solution is mechanically simpler than the above mentioned designs, where it does not need neither the tilting of the propellers nor the addition of servomotors or other mechanisms; in addition, such servomotors affect the platform efficiency due to the ensuing control latency. At the best of our knowledge, the robustness of the Y-shaped hexarotor design against propeller failure has never been experimentally tested in the static hovering sense.

Following the above discussion, the goal of the work presented in this paper is to fill this experimental gap and at the same time to provide an extensive corollary of contributions in

\footnotetext{
${ }^{1}$ https://youtu.be/cocvUrPfyfo

${ }^{2}$ https://youtu.be/HQ7wa5cBT_w?t=45
} 
this field. In particular the main contributions are summarized as follows:

1) provide a novel open source design and building of the Y-shaped hexarotor theoretical concept which has been only abstractly introduced in [7];

2) demonstrate for the first time in the literature via real experiments that the Y-shaped hexarotor is a robust platform in the static hovering sense, and therefore it could be used in safety critical environments (e.g., close to buildings and humans);

3) provide an intuitive way to understand why the collinear Y-shaped hexarotor design is robust while the collinear Star-shaped hexarotor design is not based on geometrical intuition; to provide also an intuition about the influence of parametric uncertainties on the robustness of the presented platforms;

4) carry out a systematic and extensive set of real experiments that compare the Y-shaped and Star-shaped hexarotor designs (also built in house) in the fairest way possible, both from the point of view of robustness and energy efficiency.

The rest of this paper is organized as follows: sec. II models a generic hexarotor and defines formally the Star-shaped and Y-shaped hexarotors. Sec. III defines the feasible moment set at hover of the hexarotor platform, and studies the platform's hovering and propeller robustness. Sec. IV describes the built hardware, and sec. V describes the ensuing experimental campaign. Finally, sec. VI concludes the paper.

\section{MODELING}

We consider Multi-Rotor Aerial Vehicles (MRAV) with six fixed propellers having collinear orientations. The world frame is denoted with $\mathcal{F}_{W}$, its origin with $O_{W}$ and its axes with $\left\{\boldsymbol{x}_{W}, \boldsymbol{y}_{W}, \boldsymbol{z}_{W}\right\}$ (see Fig. 1). The moving frame is denoted with $\mathcal{F}_{R}$, its origin $O_{R}$ coincides with the Center of Mass (CoM) of the platform, and its axes are denoted with $\left\{\boldsymbol{x}_{R}, \boldsymbol{y}_{R}, \boldsymbol{z}_{R}\right\}$. We denote with $\boldsymbol{p}_{R} \in \mathbb{R}^{3}$ and $\boldsymbol{R}_{R} \in S O(3)$ the position of $O_{R}$ in $\mathcal{F}_{W}$ and the rotation matrix describing the orientation of $\mathcal{F}_{R}$ with respect to (w.r.t.) $\mathcal{F}_{W}$, respectively; we further denote by $\boldsymbol{v}_{R}=\dot{\boldsymbol{p}}_{R} \in \mathbb{R}^{3}$ the linear velocity of $O_{R}$ in $\mathcal{F}_{W}$, and by $\boldsymbol{\omega}_{R}$ the angular velocity of $\mathcal{F}_{R}$ w.r.t. $\mathcal{F}_{W}$, expressed in $\mathcal{F}_{R}$. It is noted that $\dot{\boldsymbol{R}}_{R}=\boldsymbol{R}_{R}\left[\boldsymbol{\omega}_{R}\right]_{\times}$, where $[\cdot]_{\times}$denotes the map from a vector in $\mathbb{R}^{3}$ to its corresponding skew-symmetric matrix in $S O(3)$.

The platform is actuated by the set of six fixed propellers. The frame $\mathcal{F}_{p_{i}}$ is attached to the stator of the motor spinning the propeller and its origin $O_{p_{i}}$ coincides with the center of the propeller. The axes of $\mathcal{F}_{p_{i}}$ are denoted with $\left\{\boldsymbol{x}_{p_{I}} . \boldsymbol{y}_{p_{i}}, \boldsymbol{z}_{p_{I}}\right\}$, and $\boldsymbol{p}_{i} \in \mathbb{R}^{3}$ denotes the position of $O_{p_{i}}$ in $\mathcal{F}_{R}$. The $i$-th propeller rotates with a spinning rate $\omega_{i} \in \mathbb{R}$ about the $\boldsymbol{z}_{p_{i}}$ axis, creating a thrust force $\boldsymbol{f}_{i} \in \mathbb{R}^{3}$ applied at $O_{p_{i}}$ and a drag moment $\tau_{i}^{d} \in \mathbb{R}^{3}$, defined as follows:

$$
\begin{gathered}
\boldsymbol{f}_{i}=c_{f_{i}}\left\|\omega_{i}\right\| \omega_{i} \boldsymbol{z}_{p_{i}} \\
\boldsymbol{\tau}_{i}^{d}=c_{\tau_{i}}\left\|\omega_{i}\right\| \omega_{i} \boldsymbol{z}_{p_{i}}
\end{gathered}
$$
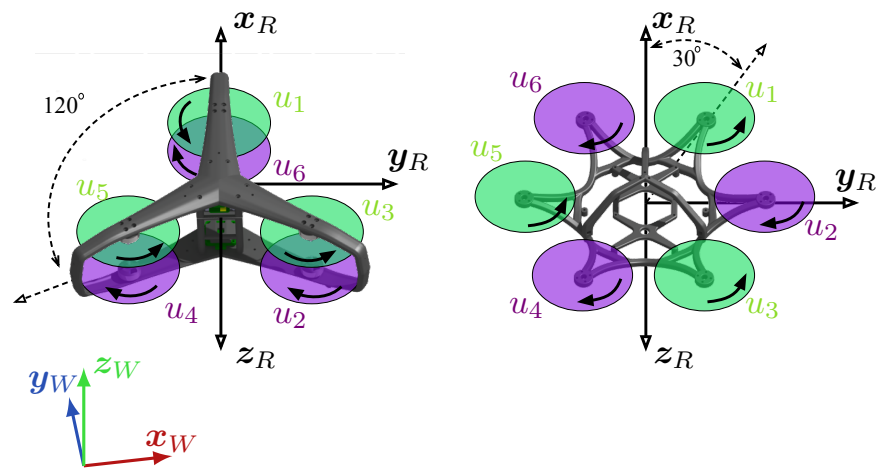

Fig. 1: System model and defined frames for Y-shaped (left) and Star-shaped (right) hexarotors.

where $c_{f_{i}} \in \mathbb{R}_{>0}$ and $c_{\tau_{i}} \in \mathbb{R}$ are the corresponding lift and drag coefficients of the corresponding propeller. The control input of the $i$-th propeller is the quantity $u_{i}=\left\|\omega_{i}\right\| \omega_{i}$.

The total resulting force $\boldsymbol{f}_{R}$ applied at $O_{R}$ and moment $\tau_{R}$ with center $O_{R}$ are expressed in $\mathcal{F}_{R}$ as follows:

$$
\begin{gathered}
\boldsymbol{f}_{R}=\sum_{i=1}^{n} c_{f_{i}} u_{i} \boldsymbol{z}_{p_{i}}, \\
\tau_{R}=\sum_{i=1}^{n}\left(\boldsymbol{\tau}_{i}^{t}+\boldsymbol{\tau}_{i}^{d}\right)=\sum_{i=1}^{n}\left(c_{f_{i}} \boldsymbol{p}_{i} \times \boldsymbol{z}_{p_{i}}+c_{\tau_{i}} \boldsymbol{z}_{p_{i}}\right) u_{i} .
\end{gathered}
$$

We assume all propellers to be identical, with three propellers rotating in one direction and the remaining three in the opposite direction; as such, $c_{f_{i}}=c_{f}$ and $c_{\tau_{i}}=\kappa_{i} c_{\tau}$, where $c_{\tau} \in \mathbb{R}^{>0}$ and $\kappa_{i}=-1(+1)$ denoting respectively a $\mathrm{CCW}(\mathrm{CW})$ direction of rotation w.r.t. $\boldsymbol{z}_{p_{i}}$. The position of $O_{p_{i}}$ in $\mathcal{F}_{R}$ is given by

$$
\boldsymbol{p}_{i}=\underbrace{\boldsymbol{R}_{z}\left(\frac{\pi}{6}+(i-1) \frac{2 \pi}{n}-\frac{1}{2}(-1)^{i} \gamma\right)}_{\boldsymbol{R}_{\gamma}(i)}\left[\begin{array}{l}
l \\
0 \\
0
\end{array}\right],
$$

with $i=1, \ldots, 6$, where $\boldsymbol{R}_{z}$ is the canonical rotation matrix about the $z$-axis. The selection of two different values for the parameter $\gamma$ allows modeling both designs considered in this work, and presented in Fig. 1, as follows:

1) Star-shape hexarotor (Fig. 1, right): is a hexarotor platform with $\gamma=0$. In this configuration, the propellers are the furthest away form each other, and thus do not overlap.

2) Y-shape hexarotor (Fig. 1, left): is a hexarotor platform with $\gamma=\frac{\pi}{3}$. In this configuration, each pair of propellers share a single rotation axis and are placed on top of each other. In order to make such design physically realizable, the pairs of coinciding propellers have to be displaced along their rotation axis. Such displacement does not affect the computation of the total force and moment because it is done along the direction of the thrust forces.

For any intermediate value of $\gamma \in\left(0, \frac{\pi}{3}\right)$ one obtains a platform that is 'in between' the two mentioned above.

\section{Feasible Moment Set}

In this paragrpah we aim to introduce the feasible moment set for a MAV, find their corresponding form for the Y-shaped 
and Star-shaped hexarotors, and study the robustness of each to propeller failure.

To start, let us use (3) and plug (5) in (4), then we can write $\boldsymbol{f}_{R}=\boldsymbol{F}_{1} \boldsymbol{u}$ and $\tau_{R}=\boldsymbol{F}_{2} \boldsymbol{u}$, where $\boldsymbol{u}=\left[\begin{array}{lll}u_{1} & \cdots & u_{6}\end{array}\right]^{\top} \in$ $\mathbb{R}^{n}$ and the force allocation matrix $\boldsymbol{F}_{1}$ and moment allocation matrix $\boldsymbol{F}_{2}$ are defined as

$$
\begin{gathered}
\boldsymbol{F}_{1}=c_{f}\left[\begin{array}{llllll}
0 & 0 & 0 & 0 & 0 & 0 \\
0 & 0 & 0 & 0 & 0 & 0 \\
1 & 1 & 1 & 1 & 1 & 1
\end{array}\right] \\
\boldsymbol{F}_{2}(\gamma)=c_{\tau}\left[\begin{array}{ccc}
r s\left(\frac{\pi}{6}-\frac{\gamma}{2}\right) & +r s\left(\frac{\pi}{2}+\frac{\gamma}{2}\right)+r s\left(\frac{5 \pi}{6}-\frac{\gamma}{2}\right) \\
-r c\left(\frac{\pi}{6}-\frac{\gamma}{2}\right) & -r c\left(\frac{\pi}{2}+\frac{\gamma}{2}\right) & -r c\left(\frac{5 \pi}{6}-\frac{\gamma}{2}\right. \\
-1 & 1 & -1 \\
r s\left(\frac{7 \pi}{6}+\frac{\gamma}{2}\right) & +r s\left(\frac{3 \pi}{2}-\frac{\gamma}{2}\right)+r s\left(\frac{11 \pi}{6}+\frac{\gamma}{2}\right) \\
-r c\left(\frac{7 \pi}{6}+\frac{\gamma}{2}\right) & -r c\left(\frac{3 \pi}{2}-\frac{\gamma}{2}\right)-r c\left(\frac{11 \pi}{6}+\frac{\gamma}{2}\right) \\
1 & -1
\end{array}\right]
\end{gathered}
$$

where $r=\left(c_{f} / c_{\tau}\right) l, s(\cdot)=\sin (\cdot)$, and $c(\cdot)=\cos (\cdot)$.

Specializing (7) for the Y-shaped $\left(\gamma=\frac{\pi}{3}\right)$ and Star-shaped $(\gamma=0)$ hexarotors one obtains:

$$
\boldsymbol{F}_{2}^{Y}=\boldsymbol{F}_{2}\left(\frac{\pi}{3}\right)=c_{\tau}\left[\begin{array}{cccccc}
0 & +r \frac{\sqrt{3}}{2} & +r \frac{\sqrt{3}}{2} & -r \frac{\sqrt{3}}{2} & -r \frac{\sqrt{3}}{2} & 0 \\
-r & +r \frac{1}{2} & +r \frac{1}{2} & +r \frac{1}{2} & +r \frac{1}{2} & -r \\
-1 & 1 & -1 & 1 & -1 & 1
\end{array}\right]
$$

$$
\boldsymbol{F}_{2}^{S}=\boldsymbol{F}_{2}(0)=c_{\tau}\left[\begin{array}{cccccc}
+r \frac{1}{2} & +r & +r \frac{1}{2} & -r \frac{1}{2} & -r & -r \frac{1}{2} \\
-r \frac{\sqrt{3}}{2} & 0 & +r \frac{\sqrt{3}}{2} & +r \frac{\sqrt{3}}{2} & 0 & -r \frac{\sqrt{3}}{2} \\
-1 & 1 & -1 & 1 & -1 & 1
\end{array}\right]
$$

Let us assume that each entry of the input $\boldsymbol{u}$ is limited between 0 and a maximum value $u_{\max }$, i.e., $\boldsymbol{u} \in$ $\mathbb{U}=X_{i=1}^{n}\left[0, u_{\max }\right]$, where $\mathbb{U}$ is the set of feasible inputs. Consequently we define the set $\mathcal{F}_{2}$ as the feasible moment set, i.e., the image set of $\mathbb{U}$ through the linear map $\boldsymbol{F}_{2}$ :

$$
\mathcal{F}_{2}(\gamma)=\left\{\tau \in \mathbb{R}^{3} \mid \exists \boldsymbol{u} \in \mathbb{U}: \tau=\boldsymbol{F}_{2}(\gamma) \boldsymbol{u}\right\}
$$

Following the above definition, we define the set of feasible inputs at hover $\mathbb{U}_{+}$as the set of control inputs that allow the compensation of the platform gravity:

$$
\mathbb{U}_{+}=\left\{\mathbf{u} \in \mathbb{R}^{3} \mid \mathbf{u} \in \mathbb{U},\left\|\boldsymbol{F}_{1} \boldsymbol{u}\right\| \geq m \mathbf{g}\right\}
$$

where $\mathbf{g} \in \mathbb{R}^{3}$ is the gravity vector w.r.t. $\mathcal{F}_{R}$; and we define the corresponding feasible moment set at hover $\mathcal{F}_{2+}$ as the image set of $\mathbb{U}_{+}$through the linear map $\mathbf{F}_{2}$. Since $\mathbb{U}_{+} \subset \mathbb{U}$, then $\mathcal{F}_{2+} \subset \mathcal{F}_{2}$.

The specialized feasible moment sets at hover for the $Y$-shaped and Star-shaped hexarotors are noted as $\mathcal{F}_{2+}^{Y}=$ $\mathcal{F}_{2+}\left(\frac{\pi}{3}\right)$ and $\mathcal{F}_{2+}^{S}=\mathcal{F}_{2+}(0)$, respectively.

The plots in the first column of Figure 2 show the feasible moment sets of the Y-shaped and Star-shaped hexarotors.

\section{A. Static Hovering}

The platform is capable of static hovering when it can reach and maintain a constant orientation and position, i.e.

$$
\dot{\boldsymbol{p}}_{R} \rightarrow \mathbf{0}, \quad \boldsymbol{\omega}_{R} \rightarrow \mathbf{0},
$$

As was explained in [7] the following conditions are needed for a platform to posses the static hovering ability

$$
\begin{aligned}
& \operatorname{rank}\left\{\boldsymbol{F}_{2}\right\}=3 \\
& \exists \boldsymbol{u} \in \operatorname{int}(\mathbb{U}) \text { s.t. }\left\{\begin{array}{rl}
\left\|\boldsymbol{F}_{1} \boldsymbol{u}\right\| & \geq m \mathbf{g} \\
\boldsymbol{F}_{2} \boldsymbol{u} & =\mathbf{0}
\end{array} .\right.
\end{aligned}
$$

Where int $(\mathbb{U})$ denotes the interior of $\mathbb{U}$.

Conditions (13) and (14) can be understood geometrically from the feasible moment set $\mathcal{F}_{2+}$ as follows:

Proposition 1. any platform for which $\mathbf{0} \in \operatorname{int}\left(\mathcal{F}_{2+}\right)$ possesses the static hovering ability.

Proof. It is a straightforward consequence of the continuity of the map $\boldsymbol{F}_{2}$. Full proof omitted for the sake of brevity.

Corollary 1. Following Prop. 1, a platform is deemed unable of static hovering if the origin is a boundary of $\mathcal{F}_{2+}$ or an external point of the set.

Lemma 1. It is easy to show that both the Y-shaped and Star-shaped hexarotors can achieve static hoverability as shown below.

Proof. $\operatorname{rank}\left\{\boldsymbol{F}_{2}^{Y}\right\}=3$ and $\operatorname{rank}\left\{\boldsymbol{F}_{2}^{S}\right\}=3$, and any input of the form $\boldsymbol{u}=\lambda \mathbf{1}=\lambda[1 \cdots 1]^{\top} \in \mathbb{R}^{n}$ with $\lambda \in\left(0, u_{\max }\right)$ belongs to $\operatorname{int}(\mathbb{U})$ and satisfies (14).

The static hovering ability of both platforms can also be seen from the feasible moment set at hover of each (Figure 2), where the origin is indeed an interior point of both $\mathcal{F}_{2+}^{S}$ and $\mathcal{F}_{2+}^{Y}$.

\section{B. Rotor Failure}

In this section we highlight the effect of propeller loss on the static hovering capability of the two hexarotors in exam. We denote by ${ }^{k} \boldsymbol{F}_{2}(\gamma)$ the moment allocation matrix $\boldsymbol{F}_{2}(\gamma)$ in which the $k$-th column has been zeroed (or, equivalently, removed). Such matrix represents the moment allocation matrix of a platform in which the $k$-th propeller does not spin anymore after a fault, i.e., $u_{k}=0$. We denote by ${ }^{k} \mathcal{F}_{2+}(\gamma)$ the feasible moment set at hover associated to ${ }^{k} \boldsymbol{F}_{2}(\gamma)$. The same specializations for the Y-shaped and Star-shaped platform apply, thus obtaining ${ }^{k} \mathcal{F}_{2+}^{Y},{ }^{k} \boldsymbol{F}_{2}^{Y},{ }^{k} \mathcal{F}_{2+}^{S},{ }^{k} \boldsymbol{F}_{2}^{S}$.

Remark 1. The feasible moment set at hover ${ }^{k} \mathcal{F}_{2+}^{S}$ for different $k$ is a rotation about the $z$-axis of ${ }^{k-1} \mathcal{F}_{2+}^{S}$, with a flip about the $(x, y)$-plane.

Proof. It is easy to see following (5) that

$$
{ }^{k} \boldsymbol{F}_{2}^{S}=\left[\begin{array}{ccc}
1 & 0 & 0 \\
0 & 1 & 0 \\
0 & 0 & -1
\end{array}\right] \boldsymbol{R}_{z}\left(\frac{\pi}{3}\right)^{k-1} \boldsymbol{F}_{2}^{S}
$$

Since all propellers are identical, then the transformation between ${ }^{k} \mathcal{F}_{2+}^{S}$ and ${ }^{k-1} \mathcal{F}_{2+}^{S}$ is the same as between ${ }^{k} \boldsymbol{F}_{2}^{S}$ and ${ }^{k-1} \boldsymbol{F}_{2}^{S}$. 


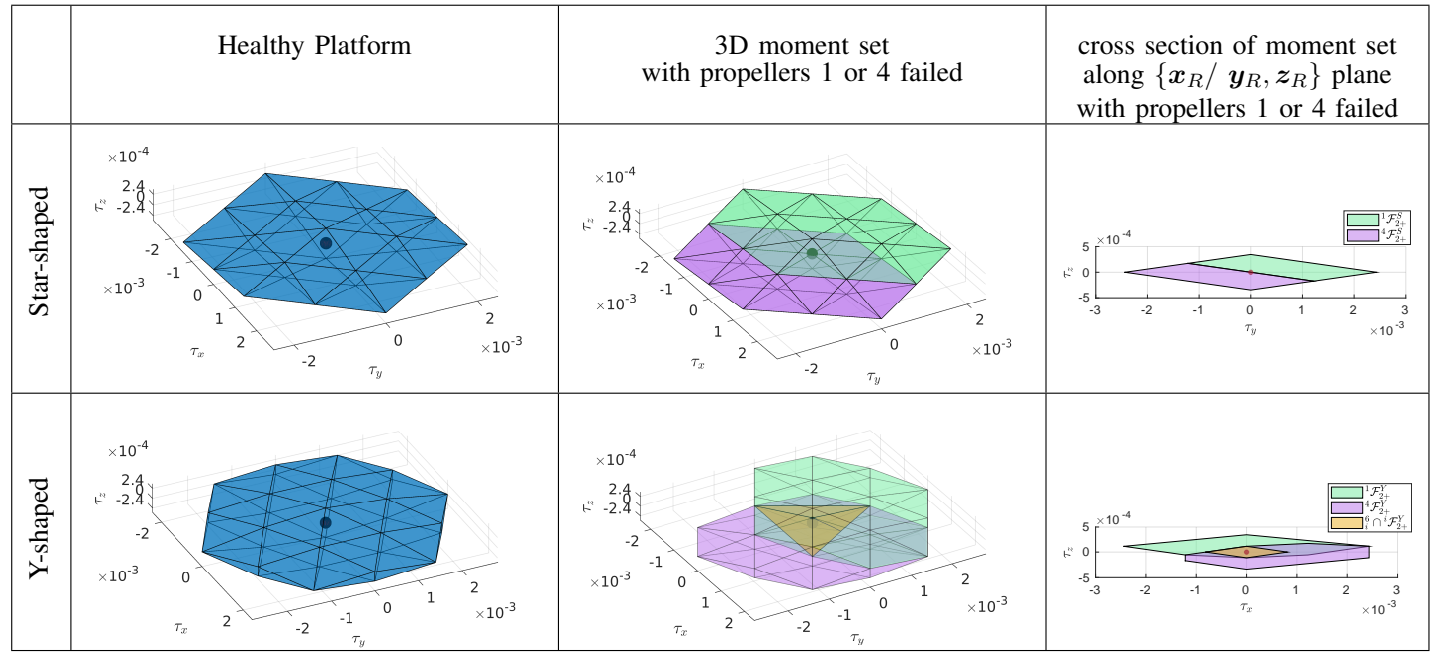

Fig. 2: Visualization of the platform feasible moment sets at hover.

It was proved in [7] that the Y-shaped hexarotor - or any collinear coplanar hexarotor with $\gamma \in\left(0, \frac{2 \pi}{3}\right)$ - is still capable of static hovering after the single loss of anyone of its propellers. On the other hand, [7] proved that the Star-shaped hexarotor $(\gamma=0)$ loses its ability to perform static hovering as it loses any of its propellers.

The static hovering ability of the two platforms can be easily understood from the geometrical viewpoint presented earlier. The vulnerability of the Star-shaped hexarotor can be seen from the corresponding feasible moment set at hover ${ }^{1} \mathcal{F}_{2+}^{S}$ shown in Figure 2, where it is clear that the origin is a point on the boundary of the presented set ; this result is similar for any ${ }^{k} \mathcal{F}_{2+}^{S}$ thanks to remark 1 . Figure 2 also shows that for any ${ }^{k} \mathcal{F}_{2+}^{Y}$, the origin of the feasible moment set at hover is an interior point, where $\mathbf{0} \in \operatorname{int}\left\{\bigcap_{k}{ }^{k} \mathcal{F}_{2+}^{Y}\right\}$.

\section{Effect of Disturbance Moment}

For any platform where static hovering is not feasible after the loss of any of its propellers, it is possible to shift the origin of the feasible moment set at hover into the interior of ${ }^{k} \mathcal{F}_{2+}$, as long as (13) and the first part of (14) are still satisfied. This can be done by adding a disturbance moment $\tau_{R}^{\text {dist }}$ such that the control moment $\tau_{R}^{c}=-\tau_{R}^{\text {dist }} \in \operatorname{int}\left({ }^{k} \mathcal{F}_{2+}\right)$.

For the Star-shaped hexarotor, for example, a disturbance moment can be obtained by shifting the CoM of the platform.

\section{Proposition 2.}

$$
\nexists \tau_{R}^{c} \in \operatorname{int}\left({ }^{k} \mathcal{F}_{2+}^{S}\right) \quad \forall k \in n
$$

i.e., it does not exist a single disturbance moment that allows to shift the origin in the interior of the feasible moment set at hover of the Star-shaped hexarotor in the case of the loss of any of the propellers.

Proof. This result is a consequence of the fact that $\operatorname{int}\left(\bigcap_{k}{ }^{k} \mathcal{F}_{2+}^{S}\right)=\emptyset$. Let us consider a moment

$$
\begin{gathered}
\tau_{R}^{1,4} \in{ }^{1} \mathcal{F}_{2}^{S} \cap{ }^{4} \mathcal{F}_{2}^{S} . \\
\tau_{R}^{1,4}=c_{\tau}\left[\begin{array}{ccccc}
+r & +r \frac{1}{2} & -r \frac{1}{2} & -r & -r \frac{1}{2} \\
0 & +r \frac{\sqrt{3}}{2} & +r \frac{\sqrt{3}}{2} & 0 & -r \frac{\sqrt{3}}{2} \\
1 & -1 & 1 & -1 & 1
\end{array}\right]\left[\begin{array}{l}
u_{2}^{1} \\
u_{3}^{1} \\
u_{4}^{1} \\
u_{5}^{1} \\
u_{6}^{1}
\end{array}\right] \\
=c_{\tau}\left[\begin{array}{ccccc}
+r \frac{1}{2} & +r & +r \frac{1}{2} & -r & -r \frac{1}{2} \\
-r \frac{\sqrt{3}}{2} & 0 & +r \frac{\sqrt{3}}{2} & 0 & -r \frac{\sqrt{3}}{2} \\
-1 & 1 & -1 & -1 & 1
\end{array}\right]\left[\begin{array}{l}
u_{1}^{4} \\
u_{2}^{4} \\
u_{3}^{4} \\
u_{5}^{4} \\
u_{6}^{4}
\end{array}\right]
\end{gathered}
$$

By simplifying then adding the first and second rows we find $u_{4}^{1}+u_{1}^{4}=-\left(u_{4}^{1}+u_{1}^{4}\right)$ to be a necessary condition for the intersection between the two sets. The only solution for the above equality is to set $u_{1}=u_{4}=0$; as such

$$
\nexists \boldsymbol{u}>0 \quad \text { s.t. } \quad \tau_{R}^{1,4} \in \operatorname{int}\left({ }^{1} \mathcal{F}_{2}^{S} \cap{ }^{4} \mathcal{F}_{2}^{S}\right)
$$

and as such, $\operatorname{int}\left({ }^{1} \mathcal{F}_{2}^{S} \cap{ }^{4} \mathcal{F}_{2}^{S}\right)=\emptyset$, and $\operatorname{int}\left(\bigcap_{k}{ }^{k} \mathcal{F}_{2}^{S}\right)=\emptyset$. Since each ${ }^{k} \mathcal{F}_{2+}^{S} \subset{ }^{k} \mathcal{F}_{2}^{S}$, then $\operatorname{int}\left(\bigcap_{k}{ }^{k} \mathcal{F}_{2+}^{S}\right)=\emptyset$ as a consequence.

The reasoning behind the above proof is also visible from the figure 2 , where it can be seen clearly that $\operatorname{int}\left({ }^{1} \mathcal{F}_{2+}^{S} \cap\right.$ $\left.{ }^{4} \mathcal{F}_{2+}^{S}\right)=\emptyset$.

\section{Effect of Model Uncertainty}

While the above modeling considers the nominal geometry of the system, manufacturing uncertainty can slightly change the actuation capabilities of the platform. More specifically, in the nominal model we consider propellers to be mounted with no tilt, i.e., $\alpha, \beta=0$. As detailed in [7], any modification in the mounting tilt can induce a stabilization of the platform. Moreover, while we consider lift and drag coefficients $c_{f}, c_{\tau}$ to be constant, they are a linear fit of the underlying nonlinear model. In addition, different propellers might have varying aerodynamic properties. Finally, in the above formulation the arm length $l$ between the CoM and each propeller is 



Fig. 3: The Y-shaped (top) and Star-shaped (bottom) hexarotor platforms built in-house for the fair comparison.

assumed constant, and the CoM is assumed to coincide with the Geometric Center (GC).

In a static hovering condition, the uncertainties mentioned above can be approximated by a lumped disturbance moment $\tau_{R}^{\text {dist; }}$; this disturbance has to be compensated so as the resultant moment applied to the platform is equal to zero.This implies that the input moment $\tau_{R}$ required for static hovering is equal to $\tau_{R}=-\tau_{R}^{\text {dist }}$ instead of zero as it would have been in the nominal case.

The presence of such disturbance $\tau_{R}^{\text {dist }}$ will practically make possible the static hovering of the Star-shaped hexarotor during the loss of some of its propellers; in particular, for any propeller loss whose feasible moment set at hover still contains the origin following the translation by $\tau_{R}^{d i s t}$. More formally, the platform can hover upon the loss of any propeller $k$ for which the following condition is verified

$$
-\tau_{R}^{d i s t} \in \operatorname{int}\left({ }^{k} \mathcal{F}_{2+}^{S}\right) \text {. }
$$

However, and as suggested in Prop. 2, for any $\tau_{R}^{\text {dist }}$ there will always exist some propellers whose loss precludes static hovering for the Star-shaped platform.

Remark 2. In the case of the Y-shaped hexarotor, let us assume that there exists a threshold moment $\tau_{R}^{\text {threshold }}$ such that

$$
\forall \tau_{R}^{\text {dist }} \leq \tau_{R}^{\text {threshold }} ;-\tau_{R}^{\text {dist }} \in \operatorname{int}\left(\mathcal{F}_{2+}^{Y}\right) .
$$

It is safe to assume that within the manufacturing and operating conditions of our platforms, $\tau_{R}^{\text {dist }} \leq \tau_{R}^{\text {threshold }}$; a similar analogy can be applied to the Y-shaped hexarotor after the failure of any of its propellers.

\section{EXPERIMENTAL PlatForm}

\section{A. Hardware: Y-shaped and Star-shaped Hexarotors}

To be able to systematically compare the Star-shaped and Y-shaped hexarotors, we design two platforms with identical components and similar properties, with the corresponding specifications shown in Table I. The two platforms, shown in Fig. 3, are built via 3D printing technology with Onyx material, and similar off-the shelf components for the propulsion system, telemetry and safety link communication. Finally, the two platforms are flown with the same autopilot and flight controller. The design of both platforms is available to the public via the following link:https://mrtbrnz.github.io/RoBust/.

\begin{tabular}{|c|c|c|c|}
\hline Specification & Star-Shape & Y-Shape & Units \\
\hline Center-Motor Distance & 0.143 & 0.130 & {$[\mathrm{~m}]$} \\
\hline Total Mass & \multicolumn{2}{|c|}{0.745} & {$[\mathrm{~kg}]$} \\
\hline Battery Capacity & \multicolumn{2}{|c|}{23.0} & [Wh] \\
\hline Flight time & 569 & 344 & [s] \\
\hline Maximum thrust & 60 & 45 & {$[\mathrm{~N}]$} \\
\hline Structure material & \multirow{2}{*}{\multicolumn{3}{|c|}{$\begin{array}{l}\text { 3D printed (Onyx composite) } \\
9 \text { pieces } 7 \text { pieces }\end{array}$}} \\
\hline Structure components & & & \\
\hline Motor \& Propeller & \multicolumn{3}{|c|}{ T-Motor F40(Kv 2400) \& 5T-5147 Prop } \\
\hline Electronic Speed Ctrl & \multicolumn{3}{|c|}{ T-Motor F45A V2.0 } \\
\hline Autopilot & \multicolumn{3}{|c|}{ Paparazzi Tawaki v1.1 } \\
\hline Communication & \multicolumn{3}{|c|}{ Xbee modem \& Futaba SBus Receiver } \\
\hline
\end{tabular}

TABLE I: Hexarotor Specifications

\section{B. Software: Paparazzi Autopilot and INDI Controller}

Throughout the flight tests, we have used the Paparazzi Autopilot system [13], an open-source autopilot that covers all three segments of the system: ground control, airborne platform control, and the communication link between the two. Paparazzi has its own flight plan language that allows the platform to follow different reference trajectories; moreover, its middle-ware allows the implementation of the desired controller on-board the platform.

The autopilot implements the INDI controller based on [14]; the controller is a robust sensor-based (measurement-based) controller which revolves around the control of the angular accelerations in an incremental way. As illustrated in [14], INDI is a robust and reliable controller, capable of dealing with strong wind perturbations and modeling inaccuracies. We refer the interested reader to the corresponding paper for more details on the control law.

While we rely on the INDI as a Fault Tolerant Control System (FTCS), we do not aim to study its advantage over other FTCS from the literature; however, we refer the curious reader to the following literature [15]-[17] for an overview of such systems and their applications.

Throughout our experimental campaign, the INDI controller runs at $500[\mathrm{~Hz}]$ while the trajectory generation is updated at a $16[\mathrm{~Hz}]$ rate.

\section{EXPERIMENTAL RESULTS}

To test the robustness and efficiency of the built platforms, an experimental campaign has been carried out at the VTO flight arena $\mathrm{a}^{3}$. The position and orientation of the vehicles are captured by the motion capture system installed in the arena.

To assess the robustness of the platforms, we introduce the following two metrics

$$
\begin{aligned}
& \frac{1}{2} m\left(\boldsymbol{e}_{\boldsymbol{p}}^{\top} \boldsymbol{e}_{\boldsymbol{p}}+\boldsymbol{v}_{R}^{\top} \boldsymbol{v}_{R}\right) \quad \text { (translation motion error) } \\
& \frac{1}{2} m \omega_{\phi}^{2} \quad \text { (rotational kinetic energy), }
\end{aligned}
$$

${ }^{3}$ https://www.enac.fr/en/drone-flight-arena-toulouse-occitanie-0 

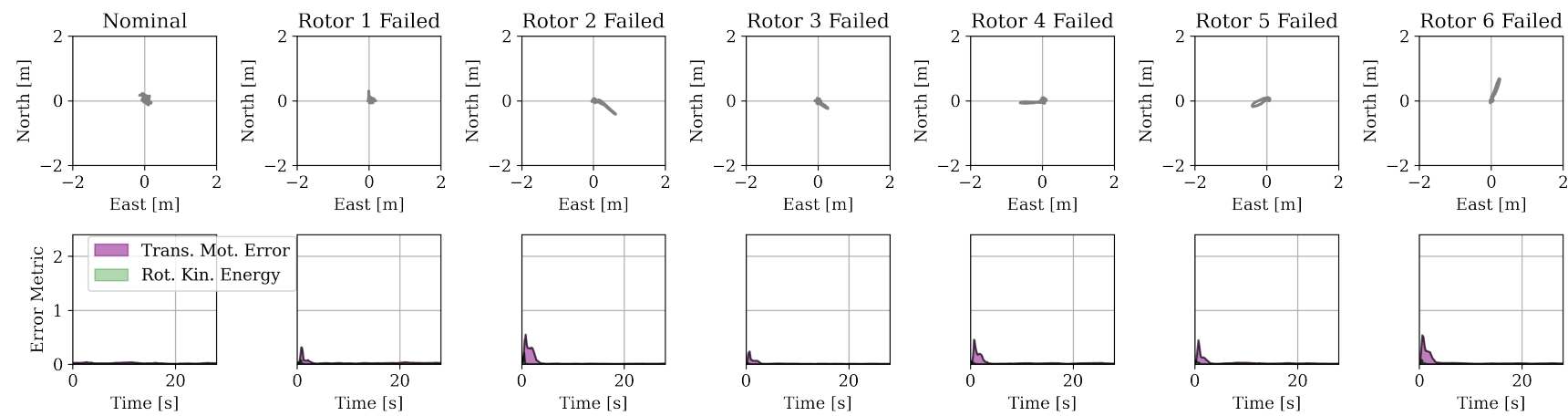

(a) Y-shaped hexarotor flights
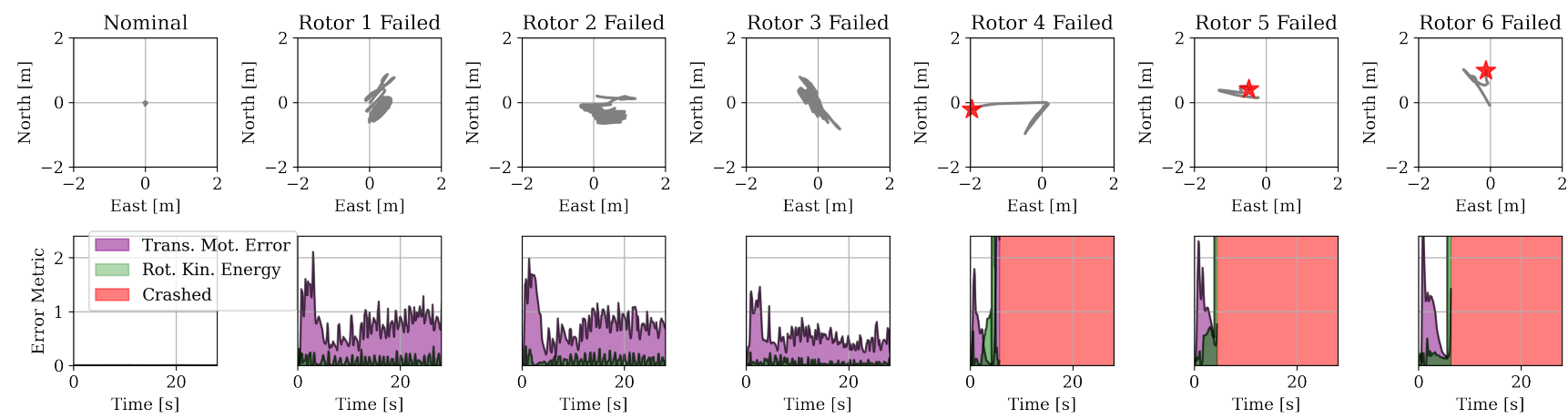

(b) Star-shaped hexarotor flight with the controller not informed of the fault
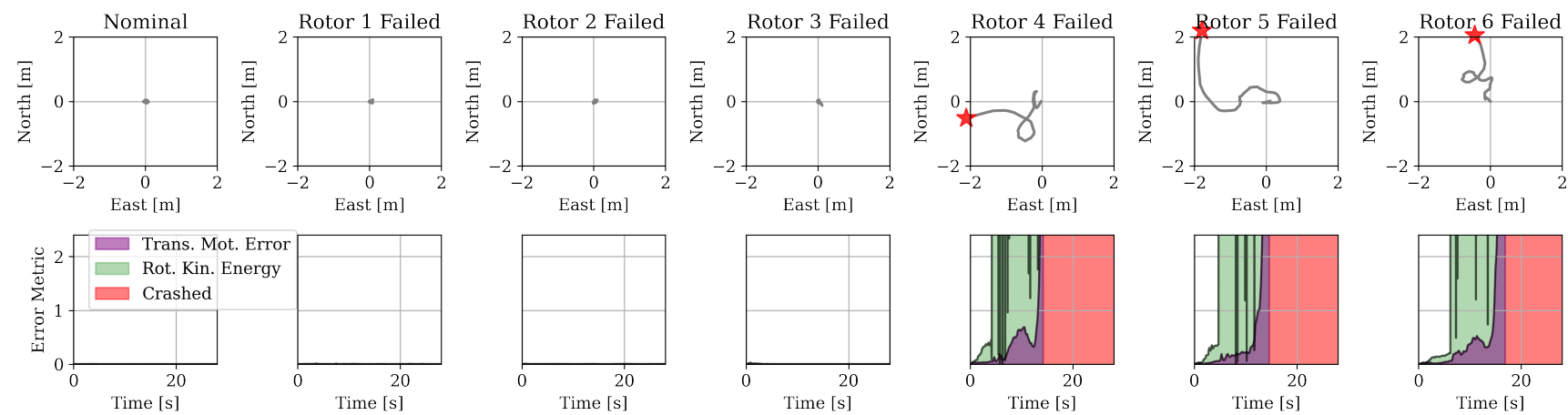

(c) Star-shaped hexarotor flight with the controller informed of the fault

Fig. 4: Each of the subfigures shows (top) the North-East projection of the flight trajectory onto the $\left(\boldsymbol{x}_{W}, \boldsymbol{y}_{W}\right)$ plane, and (bottom) the translation motion error and the rotation kinetic energy of the platform during the corresponding flight.

where $\boldsymbol{e}_{\boldsymbol{p}}=\boldsymbol{p}_{R}^{d}-\boldsymbol{p}_{R} \in \mathbb{R}^{3}$ is the positional error and $\omega_{\phi}$ is the yaw rate. It is easy to show from the underactuated dynamics and differential flatness of both vehicles that such metrics reflect the platform hovering, where each converges to zero if the platform is in static hovering, and diverges otherwise.

\section{A. Static Hovering Experimental Campaign}

To test the robustness of each platform, we synthetically induce a propeller failure while the platform is in static hovering, and assess the platform's robustness in the wake of the failure.

We note that during these experiments, and unless otherwise specified, the controller was not informed about the rotor failure, and rather attempts to fly the platform solemnly based on its measurements.

1) Static Hovering of the Y-shaped Design: First, we test the Y-shaped hexarotor to verify its robustness to propeller failures as theoretically proven in Sec. III.

Fig. 4a shows (top) the position of the Y-shaped hexarotor and (bottom) the hovering metrics of the Y-shaped hexarotor while flying with all propellers working properly and in the wake of the failure of one of each of its six propellers. As expected, the platform recovers its position after the failure of any of its propellers, with the two metrics converging to zero a few seconds after the failure.

2) Static Hovering of the Star-shaped Design: A similar experiment was conducted to test the static hovering ability of 

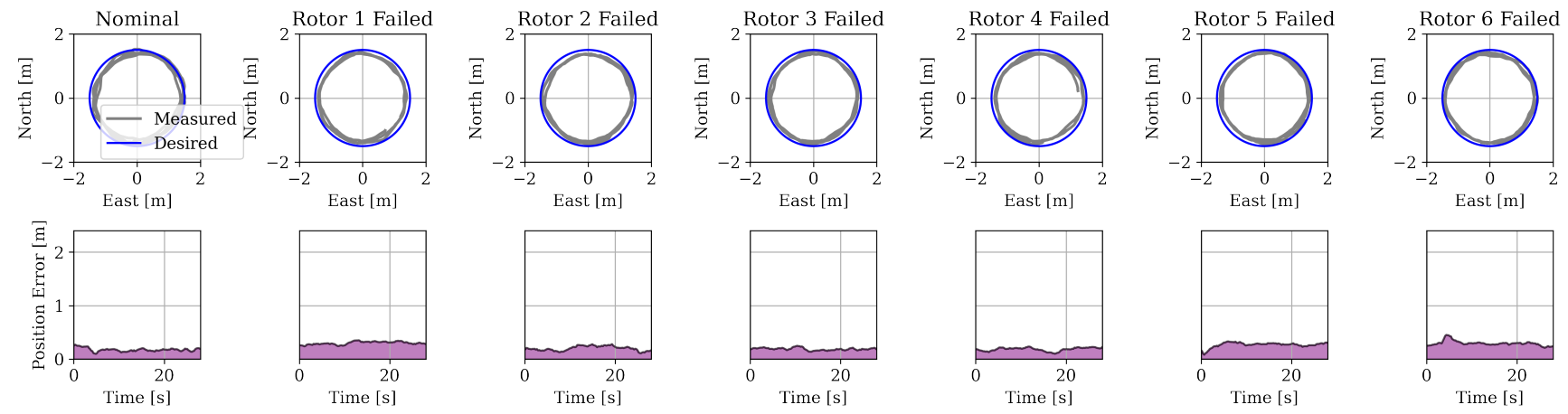

Fig. 5: Circular path following of Y-shaped hexarotor in healthy and failed rotor conditions. (Top) shows the North-East projection of the flight trajectory onto the $\left(\boldsymbol{x}_{W}, \boldsymbol{y}_{W}\right)$ plane, (bottom) shows the norm of the positional error.

the Star-shaped hexarotor.

Fig. $4 \mathrm{~b}$ shows (top) the position of the Star-shaped hexarotor and (bottom) the hovering metrics of the Star-shaped hexarotor while flying with all propellers working properly and in the wake of the failure of one of each of its six propellers. It can be seen from Fig. $4 \mathrm{~b}$ that while the healthy platform can hover normally, the Star-shaped hexarotor crashes after the failure of propellers 4-5-6. On the other hand, after the loss of propellers 1-2-3 the platform does not crash, however, it oscillates about the desired position, which can be observed in the large value of the translation motion error. The propeller failures 2 and 3, is shown in paranthesis, as they have shown different behaviour during repeatability tests, at worst ending up by crashing.

While the vulnerability of the Star-shaped hexarotor is expected (Sec. III), we repeated the above experiment for the Star-shaped hexarotor while informing the controller of the propeller fault. This is done by providing an updated actuator efficiency matrix to the controller, where the column corresponding to the failed propeller has been zeroed; this provides the controller the knowledge that the corresponding propeller has no effect on the angular rotation of the vehicle.

Fig. 4c shows the results of this experiment, where we can see that the Star-shaped hexarotor crashes right after the failure of propellers 4-5-6, while it is capable of static hovering following the failure of propellers 1-2-3. Looking deeper at the control inputs in the cases where static hovering was still possible, we can see that the informed controller was already able to generate required moments without using the corresponding propellers, and as such, the platform's performance was not affected by the corresponding failures.

These experimental results confirm the theoretical derivation presented in III-D, according to which a nominal Star-shaped hexarotor should become unstable following the failure of all its propeller, while a real Star-shaped hexarotor should become unstable following the failure of some, but not all, of its propellers, due to the manufacturing uncertainties.

Finally, we test the effect of adding a disturbance moment to the Star-shaped hexarotor on the platform's static hovering ability. The disturbance moment is induced by shifting the location of one of the platform's components in order to shift its CoM. Table II shows the static hovering ability of the
Star-shaped hexarotor following the failure of one of each of its propellers while the CoM is placed in the center of the platform, or shifted along $\boldsymbol{x}_{R}$ or $\boldsymbol{y}_{R}$. It can be seen that for each of the applied disturbance moments, and as suggested in Sec. III, the platform is vulnerable to the loss of some of its propellers, while it can successfully hover following the loss of others. Further analysis of the moment sets at hover of the platform in each of the configurations shown in Table II is presented in the attached technical report. This analysis suggests that the nominally built Star-shaped hexarotor has a manufacturing uncertainty equivalent to a shift in its $\mathrm{CoM}$ in the Front-Left direction.

\section{B. Path following after propeller failure}

To further assess the level of robustness of the Y-shaped hexarotor after propeller failure, the platform was requested to follow a circular path after the recovery from the failure of each of its six propellers. This is essential to show that the Y-shaped hexarotor is not only able to remain still but also to follow a trajectory after a failure. Figure 5 shows the results of these experiments, where it can be seen that the tracking error after propeller failure is bounded and comparable to the corresponding error of the healthy platform.

We omit the plots of the Star-shaped hexarotor circular path tracking following propeller failure, as none of the propeller failures could even satisfy the statically hovering condition.

\section{Energy consumption in healthy condition}

To assess the efficiency of the two designs, we compare the power consumption of each platform at hover. To do so, each

TABLE II: Effect of CoM shift on the robustness of the Star-shaped hexarotor

\begin{tabular}{llll}
\hline \hline Weight position & Controller & $\begin{array}{l}\text { No crash } \\
\text { when failed }\end{array}$ & $\begin{array}{l}\text { Crash when } \\
\text { failed }\end{array}$ \\
\hline Front & Not Informed & $1-2-(3)$ & $4-5-6$ \\
Back & Not Informed & $1-2-6$ & $3-4-5$ \\
Right & Not Informed & $4-5-6$ & $1-2-3$ \\
Left & Not Informed & $1-2-3$ & $4-5-6$ \\
Centered & Not Informed & $1-(2)-(3)$ & $4-5-6$ \\
Centered & Informed & $1-2-3$ & $4-5-6$ \\
\hline \hline
\end{tabular}




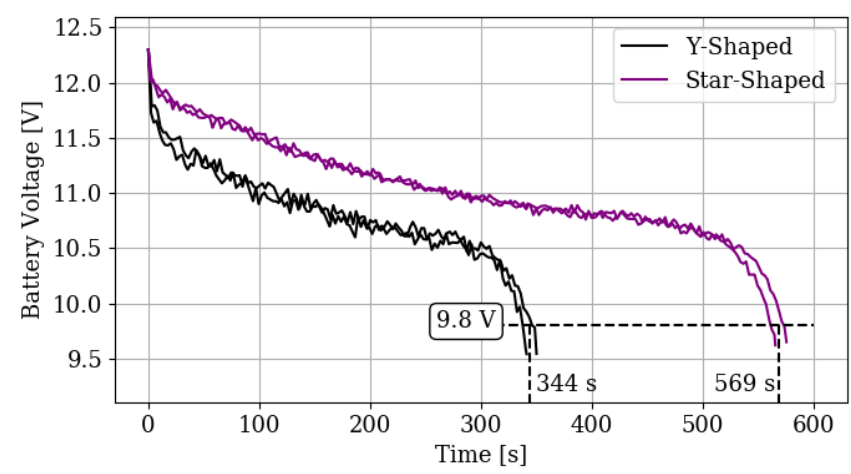

Fig. 6: Measured battery voltage during flight of Star-shaped and Y-shaped hexarotor at hover. Total flight time was recorded twice for each platform as the time for the voltage to drop from $12.6[\mathrm{~V}]$ (fully charged battery) to $9.8[\mathrm{~V}]$.

platform is flown with a fully charged battery (12.6[V]) until the battery voltage reduces to $9.8[\mathrm{~V}]$, after which the platform flight becomes unstable due to the battery's current drop. Fig. 6 shows the voltage throughout the test flights, where the flight of each platform was repeated twice. It can be seen from this figure that the flight time of the Y-shaped hexarotor is $60 \%$ of the corresponding Star-shaped flight time. In addition, the initial voltage drop of the Y-shaped hexarotor (i.e., the voltage drop required for take off) is higher than the corresponding drop in the case of the Star-shaped hexarotor, which suggests a higher drawn current at hover.

The reduction in efficiency is expected to be caused in part to the interaction between the co-axial propellers and in part to the increased interaction between the flow of the propellers with the arms connecting the propellers to the platform, given that the arms of the Y-shaped hexarotor are made wider than those of the Star-shaped hexarotor to gain the required structural robustness.

\section{CONCLUSIONS}

In this work we introduced an open source design of a Y-shaped and Star-shaped hexarotor. The two designs are built with identical components and similar properties to systematically compare the abilities of each. The two platforms reply on the INDI controller to fly robustly even after the failure of any(some) of their propellers respectively.

In addition, we introduce an intuitive geometrical interpretation of the platforms' static hovering ability. Following this geometric interpretation, we show the vulnerability of the Star-shaped hexarotor to the single failure of some of its propellers and the robustness of the Y-shaped hexarotor to the single failure of any of its propellers.

The static hovering of the two designs is further studied via an extensive experimental campaign that validates the theoretical hypotheses. In addition, their respective efficiency was tested comparing the power consumption of each.

Following the above analysis, we can clearly see that while the Y-shaped hexarotor is robust to the failure of any propeller, it is less efficient than the Star-shaped design. On the other hand, the Star-shaped design is a more efficient design, while it is vulnerable to the failure of some of its propellers.

The study of a platform that can benefit from the efficiency and robustness of each of the two designs is an interesting research line that is left as a future work. 


\section{APPENDIX A}

\section{Detailed Moment Set Analysis During Failure and} MODIFIED CENTER OF MASS

This technical report analyzes the moment set at hover of the Star-shaped hexarotor platform following the failure of each propeller, with different CoM configurations, in an aim to understand the underlying causes of the ensuing platform crashes.

As was discussed in [18], the behaviour of the Star-shaped hexarotor is a peculiar one following the failure of different propellers as illustrated theoretically in III-C and III-D, and experimentally in V-A2 from [18]. Due to uncertainties in manufacturing and component placement, it is very difficult to have a perfectly symmetric experimental platform, making it a hard job to predict which propeller failure will lead to an inability of static hovering. As such, the main investigation in the conducted experiments was to observe if there will be at least one propeller failure leading to an incapability of static hovering, for different platform configurations (different CoM configurations, controller informed or not).

As proven in [7] and discussed in III-B (from [18]), for a perfectly manufactured Star-Shaped hexarotor, failure of any of its propellers will result in the loss of static hovering capability since the origin resides at the boundary of the feasible moment set as shown in Figure 2 (from [18]). However during our experiments, we have observed that for some of the rotor failures, the Star-Shaped hexarotor is capable of static hovering, while other propeller failures lead to crashes. This discrepancy is expected to be due to the aforementioned manufacturing uncertainties (III-D from [18]). In fact, even small construction inaccuracies may cause the origin to be inside or outside the moment set depending on the failed propeller.

In order to point out this effect and further analyze the experimental results given in Table II (from [18]), additional numerical calculations are shown in Figure 7 for each propeller failure while changing CoM location systematically to right, left, front and back positions ( $1 \mathrm{~cm}$ in each direction).

Figure 7 shows the expected moment set at hover for different CoM positions at $\tau_{z}=0$ plane for each rotor failure case. In the case of no external disturbance, and no maneuver requirement, statically hovering condition requires the origin point to be an interior point of the moment set (purple area).

The projection of the origin on $\tau_{z}=0$ plane is shown in all plots i) in green for experimental flights where the platform was capable of static hovering, and ii) in red for experimental flights where the platform crashed following the propeller failure. It can be seen from the corresponding moment sets that for flights where the platform crashed, the origin is at best a boundary point of the moment set at hover, while it is an interior point in the case of flights where static hovering was still possible after the corresponding propeller failure. The only exception is the Front Heavy - Rotor-1 failure case, where the platform managed to fly after the propeller failure, despite the fact that the origin slightly surpasses the moment set boundary. While in the presented moment sets, we are assuming that the disturbance caused by the placement of the CoM to be the only source of disturbance, manufacturing imperfections are still present, and could in some cases have a larger effect than the former disturbance. As such, we believe these imperfections, as mentioned in III-D (from [18]), to be the source of this discrepancy.

In the case of centered CoM, shown in Figure 8, the origin is a boundary point of each of the corresponding moment sets at hover. While this should lead the vehicle to fail static hovering for any of the rotor failures, it can be seen from the experiments that the first three rotor failures did not induce a platform crash, while the other failures did.

As such, and following the two experiments, we can estimate that our experimental platform has a slight CoM shift towards front and left as it presents the same crash patterns as the platform with the corresponding CoM shift.

\section{REFERENCES}

[1] R. Rashad, J. Goerres, R. Aarts, J. Engelen, and S. Stramigioli, "Fully actuated multirotor uavs: A literature review," IEEE Robotics \& Automation Magazine, 2020.

[2] T. Tomić, P. Lutz, K. Schmid, A. Mathers, and S. Haddadin, "Simultaneous contact and aerodynamic force estimation (s-cafe) for aerial robots,' The International Journal of Robotics Research, vol. 39, no. 6, pp. 688728, 2020.

[3] M. Tognon, R. Alami, and B. Siciliano, "Physical human-robot interaction with a tethered aerial vehicle: Application to a force-based human guiding problem," arXiv preprint arXiv:2005.06760, 2020.

[4] D. Tezza and M. Andujar, "The state-of-the-art of human-drone interaction: A survey," IEEE Access, vol. 7, pp. 167438-167454, 2019.

[5] M. Hamandi, F. Usai, Q. Sablé, N. Staub, M. Tognon, and A. Franchi, "Survey on Aerial Multirotor Design: a Taxonomy Based on Input Allocation," Jan. 2020, working paper or preprint. [Online]. Available: https://hal.archives-ouvertes.fr/hal-02433405

[6] M. W. Mueller and R. D'Andrea, "Relaxed hover solutions for multicopters: Application to algorithmic redundancy and novel vehicles," The International Journal of Robotics Research, vol. 35, no. 8, pp. 873-889, 2016.

[7] G. Michieletto, M. Ryll, and A. Franchi, "Fundamental actuation properties of multi-rotors: Force-moment decoupling and fail-safe robustness," IEEE Trans. on Robotics, vol. 34, no. 3, pp. 702-715, 2018.

[8] M. Kamel, K. Alexis, M. Achtelik, and R. Siegwart, "Fast nonlinear model predictive control for multicopter attitude tracking on $\mathrm{SO}(3)$," in IEEE Conf. on Control Applications, Sydney, Australia, Sep. 2015.

[9] D. Bicego, J. Mazzetto, M. Farina, R. Carli, and A. Franchi, "Nonlinear model predictive control with enhanced actuator model for multi-rotor aerial vehicles with generic designs," Journal of Intelligent \& Robotics Systems, 2020.

[10] J. I. Giribet, R. S. Sanchez-Pena, and A. S. Ghersin, "Analysis and design of a tilted rotor hexacopter for fault tolerance," IEEE Trans. on Aerospace and Electronic System, vol. 52, no. 4, pp. 1555-1567, 2016.

[11] G. Michieletto, M. Ryll, and A. Franchi, "Control of statically hoverable multi-rotor aerial vehicles and application to rotor-failure robustness for hexarotors," in 2017 IEEE Int. Conf. on Robotics and Automation, Singapore, May 2017, pp. 2747-2752.

[12] C. Pose, J. Giribet, and I. Mas, "Fault tolerance analysis for a class of reconfigurable aerial hexarotor vehicles," IEEE/ASME Transactions on Mechatronics, 2020.

[13] G. Hattenberger, M. Bronz, and M. Gorraz, "Using the paparazzi uav system for scientific research," 2014.

[14] J. Smeur, G. de Croon, and Q. Chu, "Cascaded incremental nonlinear dynamic inversion for mav disturbance rejection," Control Engineering Practice, vol. 73, pp. 79-90, 2018.

[15] Y. Zhang, A. Chamseddine, C. A. Rabbath, B. W. Gordon, C.-Y. Su, S. Rakheja, C. Fulford, J. Apkarian, and P. Gosselin, "Development of advanced fdd and ftc techniques with application to an unmanned quadrotor helicopter testbed," Journal of the Franklin Institute, vol. 350, no. 9, pp. 2396-2422, 2013.

[16] M. W. Mueller and R. D'Andrea, "Stability and control of a quadrocopter despite the complete loss of one, two, or three propellers," in 2014 IEEE international conference on robotics and automation (ICRA). IEEE, 2014, pp. 45-52. 

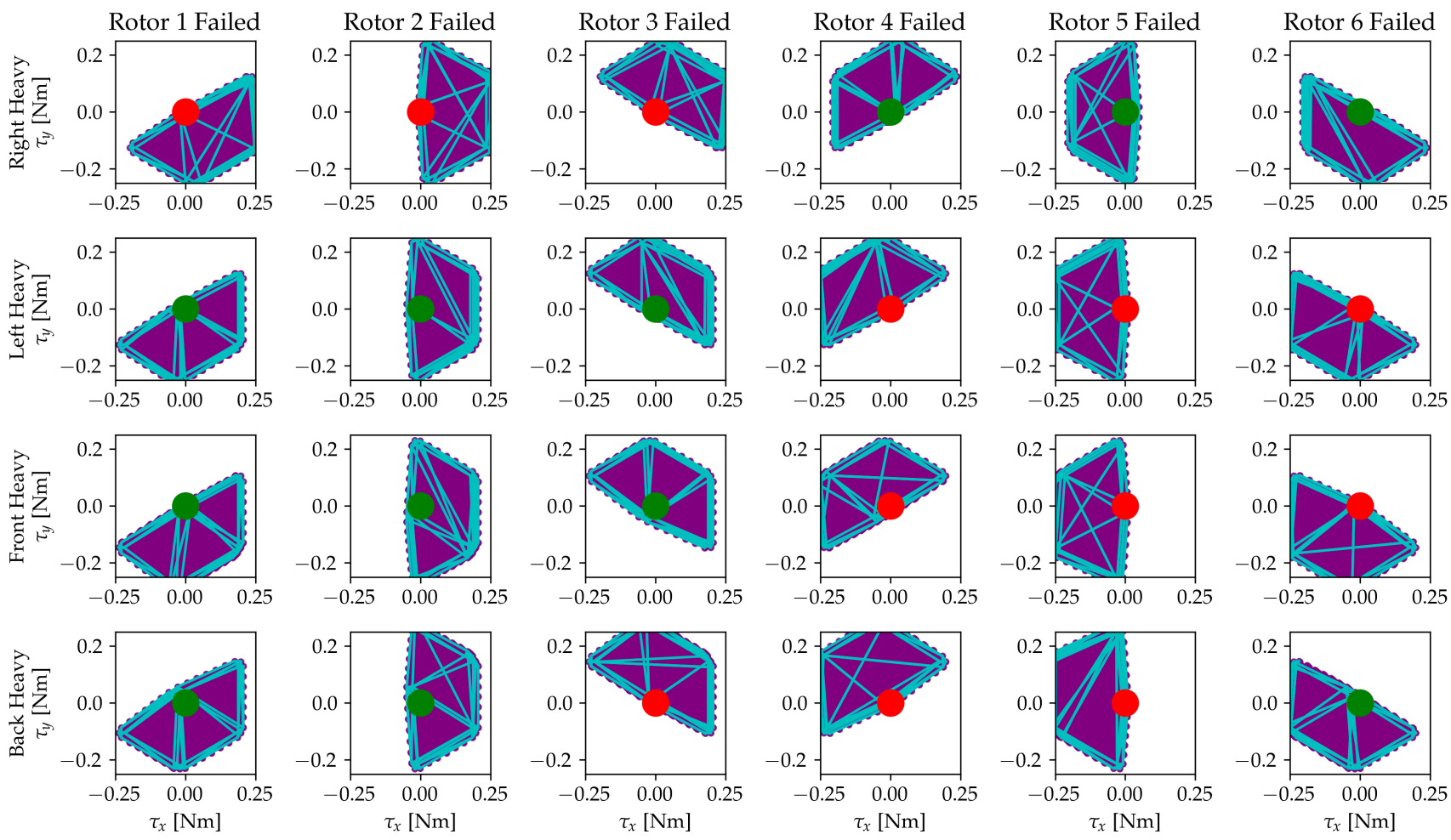

Fig. 7: Visualization of the cross sections at the $\tau_{z}=0$ plane of the platform's feasible moment sets at hover for different propeller failures, and different CoM configurations. The origin of the feasible moment sets at hover is shown in green for flights where the platform was capable of static hovering after the propeller failure, and in red when the platform crashed following the propeller failure in experiments.
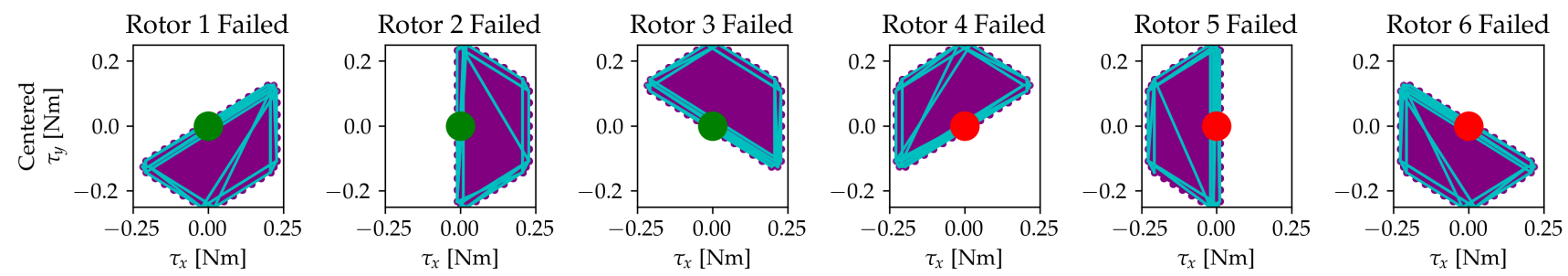

Fig. 8: Visualization of the cross sections at the $\tau_{z}=0$ plane of the platform's feasible moment sets at hover for different propeller failures when the CoM is perfectly centered. The origin of the feasible moment sets at hover is shown in green for flights where the platform was capable of static hovering after the propeller failure, and in red when the platform crashed following the propeller failure in experiments.

[17] D. Scaramuzza, M. C. Achtelik, L. Doitsidis, F. Friedrich, E. Kosmatopoulos, A. Martinelli, M. W. Achtelik, M. Chli, S. Chatzichristofis, L. Kneip et al., "Vision-controlled micro flying robots: from system design to autonomous navigation and mapping in gps-denied environments," IEEE Robotics \& Automation Magazine, vol. 21, no. 3, pp. 26-40, 2014.

[18] E. Baskaya, M. Hamandi, M. Bronz, and A. Franchi, "A novel robust hexarotor capable of static hovering in presence of propeller failure," In revision to IEEE Robotics and Automation Letters, 2021. 